\title{
Reabilitação auditiva por aparelho de Amplificação Sonora Individual (AASI) em centro especializado do SUS de Salvador-Bahia
}

\author{
Auditory rehabilitation by hearing aid in SUS specialized center of Salvador-Bahia \\ Edinaide Lopes da Silva Lins ${ }^{1}$, Fernando Pena Gaspar Sobrinho ${ }^{*}$
}

\begin{abstract}
${ }^{1}$ Fonoaudióloga, graduada pela Universidade Estadual da Bahia(UNEB); ${ }^{2}$ Doutor em Medicina pela Universidade Federal da Bahia. Professor adjunto do Departamento Ciências da Vida da UNEB.
\end{abstract}

\begin{abstract}
Resumo
Introdução: a hipoacusia permanente é um problema comum. Sua abordagem inclui reabilitação através de prótese auditiva. 0 Sistema Único de Saúde oferece reabilitação auditiva à população brasileira. Objetivo: descrever o perfil do usuário de prótese auditiva do Setor Auditivo do Centro de Prevenção e Reabilitação da Pessoa com Deficiência, da Bahia. Metodologia: estudo observacional de corte transversal, com consulta a 115 prontuários de adultos com indicação de prótese auditiva. Utilizou-se instrumento de coleta de dados com as seguintes variáveis: características demográficas, presença de queixas à anamnese (hipoacusia, zumbido e vertigem), achados à otoscopia, e tipos de: perda auditiva, adaptação, aparelho auditivo, molde, e de material utilizado na confecção do molde. Obteve-se de estatística descritiva: frequência, média, mediana e porcentagem. Resultados: predominaram o sexo feminino (63/54,7\%) e idosos (74/64,3\%). A queixa otológica mais comum foi a hipoacusia $(114 / 99,1 \%)$, seguida de zumbido $(67 / 58,2 \%)$ e tontura $(41 / 35,7 \%)$. Otoscopia normal em $(223 / 97,0 \%)$ das orelhas. Encontraram-se perdas auditivas sensorioneural $(160 / 69,7 \%)$, mista $(62 / 27,0 \%)$ e condutiva $(07 / 3,0 \%)$. A adaptação binaural e o aparelho retroauricular foram os mais utilizados $(111 / 96,5 \%)$ e $(220 / 95,7 \%)$, respectivamente, secundado por intra-auricular $(04 / 1,7 \%)$ e intracanal $(0,2 / 0,9 \%)$. Os moldes foram tipo concha $(159 / 69,1 \%)$, aberto $(34 / 14,8 \%)$, e canal $(33 / 14,4 \%)$, e o material mais usado para confecção do molde foi o silicone (181/78,8\%), quando comparado ao acrílico (45/19,2\%). Conclusão: maior prevalência de idosos e mulheres; expressiva presença de queixas otológicas associadas à perda auditiva; maior demanda para adaptação binaural por aparelho tipo retroauricular com molde concha de silicone.
\end{abstract}

Palavras-chave: Hipoacusia. Aparelho Auditivo. Reabilitação. Audição. Sistema Único de Saúde.

\begin{abstract}
Introduction: permanent hearing loss is a common problem. Its approach includes exercise therapy through hearing aids. The Unified Health System offers auditory rehabilitation to the Brazilian population. Objective: describe the auditory rehabilitation profile of the Auditory Sector of Center for Prevention and Rehabilitation of the Disabled of Bahia. Methodology: this is a cross-sectional observational study. We consulted 115 medical records of adults with hearing aids indication. We used a data collection instrument with the following variables: demographic characteristics, presence of complaints to anamnesis (hypoacusis, tinnitus and vertigo), otoscopy findings (except for irrelevant findings), and types of hearing loss, adaptation, hearing aids, mold, and material used to make the mold. Descriptive statistics were obtained: frequency, average, median and percentage. Results: there was a female $(63 / 54,7 \%)$ and elderly $(74 / 64,3 \%)$ predominance. Hypoacusis was the most common otiologic complaint (114/99.1\%), followed by tinnitus $(67 / 58,2 \%)$ and dizziness $(41 / 35,7 \%)$. Otoscopy was normal in $(223 / 97,0 \%)$ of the ears. The types of hearing loss found were sensorineural (160/69,7\%), mixed (62/27,0\%) and conductive (07/3,0\%). Bilateral rehabilitation and Behind-The-Ear/BTE type apparatus were the most used (111/96,5\%) and (220/95.7\%), respectively, followed by In-The-Ear/ITE (04/1,7\%) and In-The-Canal) ITC $(02 / 0,9 \%)$. The molds were of the shell type $(159 / 69,1 \%)$, open $(34 / 14,8 \%)$, and canal $(33 / 14,4 \%)$, and the most used material to make it was silicone $(181 / 78,8 \%)$ when compared to acrylic $(45 / 19,2 \%)$. Conclusion: there was a high prevalence of elderly and female; expressive presence of otologic complaints associated with hearing loss; and a greater demand for bilateral rehabilitation by Behind-The-Ear type apparatus with shell type mold made with silicone.
\end{abstract}

Keywords: Hearing loss. Hearing aid. Rehabilitation. Hearing. Unified Health System

\section{INTRODUÇÃO}

A capacidade auditiva compreende um dos sentidos fundamentais na constituição da relação do indivíduo com a sociedade e com o ambiente. A redução da acui-

Correspondente/Corresponding: *Fernando Pena Gaspar Sobrinho End.: Universidade do Estado da Bahia, Depto. de Ciências da Vida, Área de Ciências Biológicas, Rua Silveira Martins, 2555, Cabula. Salvador, Bahia. Tel.:(71) 31175344 - E-mail: gaspar500@gmail.com dade auditiva, chamada perda auditiva (PA) ou hipoacusia, é uma condição patológica de privação sensorial decorrente de diversas etiologias, em qualquer faixa etária, afetando sobretudo idosos, quando é chamada presbiacusia (GÂNDARA; ALVES, 2017). Há limitação do acesso aos sons ambientais, e, igualmente, à linguagem oral, tornando difícil a compreensão dessas mensagens sonoras, por afetar os processos perceptivos, como atenção e discriminação. 
Segundo lório (2015), a perda auditiva é a privação sensorial que produz maior impacto no processo da comunicação. Pode haver limitação na realização de atividades, compromete um mecanismo de defesa, útil para a sobrevivência, na medida em que impossibilita ouvir sons de alerta, e restringe a participação do deficiente auditivo em situações de vida diária. Suas consequências negativas ultrapassam a restrição sonora. Destarte, a perda auditiva é um problema de saúde pública devido às múltiplas consequências e à sua significativa prevalência e impacto socioeconômico (FERRITE et al., 2011).

Para o IBGE (2017), há aumento da população de idosos no Brasil, bem como da prevalência do sexo feminino nesta faixa etária. Cordeiro (2014) encontrou predomínio de perda auditiva em pacientes idosos e do sexo feminino num estudo realizado em Salvador, Bahia. Em adição, outros sintomas estão associados à perda auditiva, como o zumbido, que pode ser a primeira manifestação de doença otológica, bem como a tontura (DORIGUETO et al., 2017). Por outro lado, a reabilitação auditiva e os processos diagnósticos e terapêuticos envolvidos têm um custo relativamente elevado, exigindo políticas públicas voltadas para essa população, que devem levar em consideração as mudanças demográficas e os avanços científico-tecnológicos.

O Ministério da Saúde (MS) instituiu a Política Nacional de Atenção à Saúde Auditiva (PNASA) em 2004, sob Portaria no 2.073 , na qual propôs a organização, segundo os princípios do Sistema Único de Saúde (SUS), de oferta da atenção à saúde auditiva em uma rede hierarquizada, regionalizada e integrada, visando o diagnóstico e a reabilitação auditiva, a promoção, a proteção, e a terapia fonoaudiológica de adultos e crianças. Para sua efetivação operacional, estabeleceu a Portaria SAS/MS no 587, de 7 de outubro de 2004, determinando que as Secretarias de Saúde dos Estados adotassem as providências necessárias à organização e implantação das Redes Estaduais de Atenção à Saúde Auditiva.

Os Centros Especializados em Reabilitação (CER) de nível III, a exemplo do Centro Estadual de Prevenção e Reabilitação de Deficiências (CEPRED), em Salvador, Bahia, no rol de suas atribuições, é, atualmente, uma rede de cuidados. Dentre os serviços prestados, está a distribuição gratuita de aparelhos auditivos (próteses auditivas) custeados pelo SUS, proporcionando assistência e acessibilidade às pessoas com perda auditiva (SILVA et al., 2010).

O Aparelho de Amplificação Sonora Individual (AASI), conhecido como prótese auditiva, é um mini-amplificador eletrônico cujo propósito é compensar as limitações e impedimentos provocados pela diminuição da acuidade auditiva, melhorando a compreensão da fala em várias situações. Sua finalidade é maximizar o resíduo auditivo através da captura dos sons do ambiente e de fala, com aumento da intensidade sonora (PEREIRA, 2015). Isso torna a audição residual mais funcional e proporciona melhor qualidade de vida e proteção ao usuário.
A prescrição do AASI é um trabalho multidisciplinar que envolve, principalmente, as áreas da otorrinolaringologia, de onde provém o diagnóstico otológico, subsidiado pelo diagnóstico audiológico, e da fonoaudiologia, com todo o processo de reabilitação. $O$ audiologista atua desde a avaliação audiológica diagnóstica, até a pré-moldagem auricular, escolha do tipo (concha, aberto e canal) e o material do molde auricular (silicone ou acrílico), seleção do AASI adequado (retroauricular ou intra-aural) através de teste, e a adaptação do mesmo. Nesta fase, que também considera as condições estéticas e a expectativa do paciente, se confirma a indicação da orelha a ser protetizada, unilateral ou bilateral (PAGNOSSIM et al., 2010; PIZA, 2017; TEIXEIRA; GARCEZ, 2015).

Conhecer o usuário de AASI de uma rede pública instituída ajudará a compreender como as demandas estão estruturadas, o perfil do usuário e as características de seus insumos. Tal conhecimento poderá ser útil tanto aos profissionais da assistência quanto aos gestores, visto que informações dessa natureza são de grande valia para o planejamento prático e técnico, e para subsidiar tomadas de decisões que fomentem a promoção da saúde auditiva. Ressalte-se ainda que áreas envolvendo tecnologias necessitam de estudos para avaliar a política aplicada e sua efetividade, permitindo a otimização de verbas. Deste modo, o presente estudo tem como objetivo descrever o perfil demográfico, clínico e de reabilitação de pacientes usuários de AASI atendidos num CER de Salvador, Bahia.

\section{METODOLOGIA}

Foi realizado um estudo observacional do tipo corte transversal, através de consulta a registros de prontuários de pacientes consecutivamente atendidos no CEPRED entre os meses de maio e julho de 2016. Este estudo foi aprovado pelo Comitê de Ética em Pesquisa (CEP) da Universidade do Estado da Bahia (UNEB), sob o Parecer no 1.428.608, em 29 de Fevereiro de 2016.

Esta pesquisa deriva de um estudo mais amplo intitulado "Frequência de Queixas Otológicas em Usuários do Setor de Reabilitação Auditiva de um Centro Especializado em Reabilitação". Este estudo foi realizado a partir de prontuários de pacientes que receberam AASI concedido até um ano anterior ao período da coleta, e incluiu tanto primeiras concessões quanto substituições de AASI. Os pacientes assinaram o Termo de Consentimento Livre Esclarecido (TCLE). Esses prontuários, selecionados por conveniência, incluíam registros da avaliação audiológica completa realizada pelos profissionais fonoaudiólogos e avaliação médica pelo otorrinolaringologista do Setor de Audiologia do CEPRED.

Foram incluídos prontuários com diagnóstico audiológico de perda auditiva de pacientes com idade de 18 anos ou mais, na medida em que esses pacientes já possuem autonomia e responsabilidades sobre suas condutas frente ao atendimento médico e fonoaudiológico, ou com qualquer demanda de reabilitação auditiva por 
AASI. Foram excluídos os prontuários com informações incompletas.

As informações coletadas dos prontuários foram registradas em um instrumento de coleta de dados (Apêndice A) elaborado pelos próprios pesquisadores e contendo as seguintes variáveis: dados demográficos (gênero e faixa etária), queixas otológicas (hipoacusia, zumbido e tontura), achados à otoscopia (classificada como normal ou anormal, exceto a presença cera, otites externas e alterações menores), tipo de perda auditiva (sensorioneural, condutiva e/ou mista), se perda uni ou bilateral, tipo do AASI indicado (intra-aurais - intra-auricular, intracanal ou microcanal - ou retroauricular), lateralidade da protetização (uni ou bilateral), tipo de tecnologia (digital, hibrida ou analógica), tipo de molde (concha, aberto e canal) e materiais (silicone ou acrílico) de confecção do molde auricular. Não foram pesquisados o grau das perdas auditivas, bem como os resultados da logoaudiometria e da imitanciometria. As queixas auditivas foram computadas levando-se em consideração a frequência de citação dos sintomas, ao contrário das demais informações otológicas e audiológicas, que se deram pelo quantitativo de orelhas, sem distinção entre orelha esquerda ou direita. Para analisar a variável faixa etária, a amostra foi classificada em três categorias: adulto-jovem, de 18 a 40 anos; adulto, de 41 a 59 anos; e idoso, a partir de 60 anos.

Os dados foram organizados e analisados por meio do programa estatístico SPSS, versão 16 . Foi realizada estatística descritiva com medidas de frequência, média, mediana e porcentagem.

\section{RESULTADOS}

Foram analisados 115 prontuários. Sessenta e três pacientes foram do sexo feminino $(54,7 \%)$ e 52 do sexo masculino (45,3\%). A idade variou de 18 a 94 anos, com maior prevalência de idosos (Gráfico 1) sendo os valores da média e da mediana de 63,5 e 67 anos, respectivamente. $E$, entre os sexos, a média e a mediana foram 63 e 64 anos para o sexo feminino, e 63,5 e 69 anos para o sexo masculino.

Gráfico 1 - Faixa etária dos pacientes analisados.

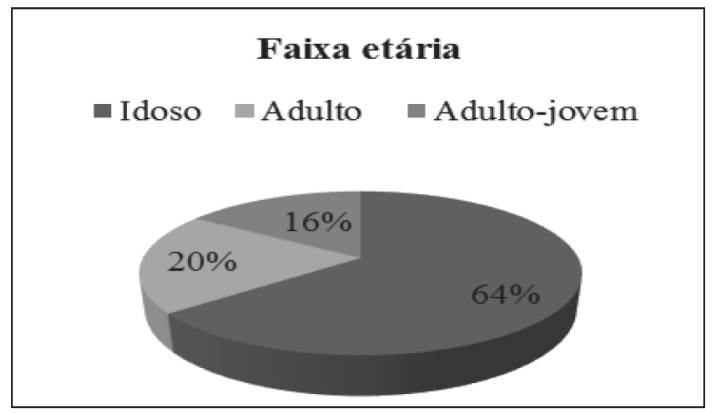

Fonte: Elaboração própria
A queixa mais comum foi de hipoacusia, seguida por zumbido e tontura (Gráfico 2). Um paciente $(0,9 \%)$ queixou-se apenas de zumbido, embora a audiometria indicasse perda auditiva. A perda auditiva bilateral foi encontrada em $114(99,1 \%)$ pacientes, enquanto a unilateral apresentou-se apenas em um (0,9\%). 0 tipo mais frequente foi sensorioneural (Gráfico 3). Levando-se em conta idade e perda auditiva, a predominância do tipo sensorioneural bilateral foi de $75,6 \%$ para pacientes com 60 anos ou mais.

Gráfico 2 - Queixas otológicas citadas pelos pacientes durante anamnese.

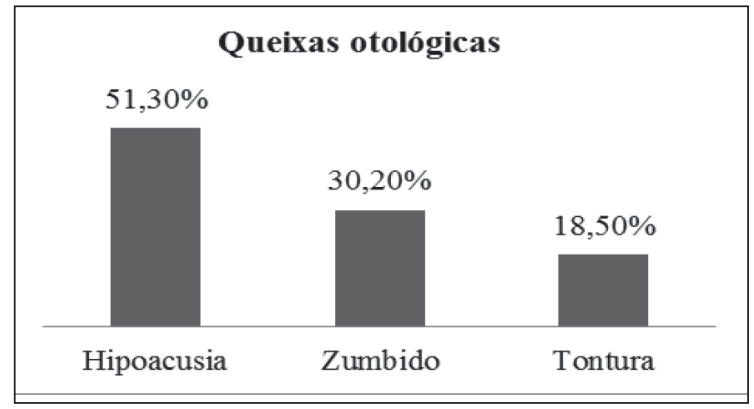

Fonte: Elaboração própria

Gráfico 3 - Frequência dos tipos de perda auditiva, por orelha, com base na audiometria.

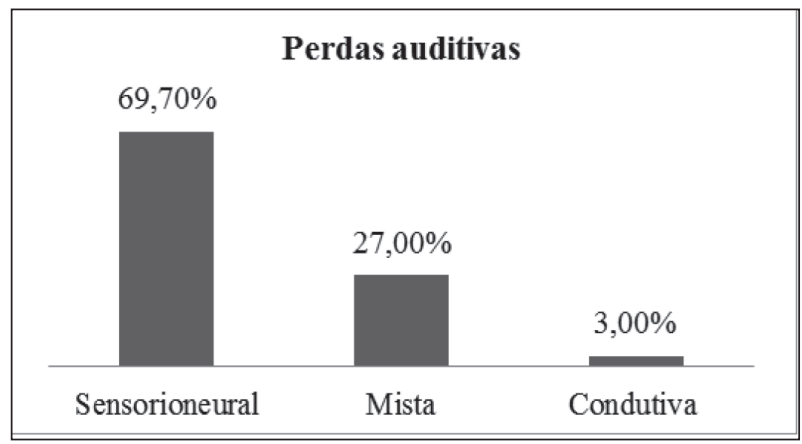

* Um paciente apresentou estenose unilateral de MAE, o que equivale a $0,30 \%$ de perda auditiva não classificada.

Fonte: Elaboração própria

A otoscopia com características normais obteve registro expressivo entre indivíduos analisados (Gráfico 4). Dentre as anormalidades observadas à otoscopia, quatro pacientes apresentavam membranas timpânicas atelectásicas, enquanto dois pacientes tinham perfurações nas membranas. Foi observado um caso de estenose unilateral de meato acústico externo. 
Gráfico 4 - Achados à otoscopia, por orelha examinada.

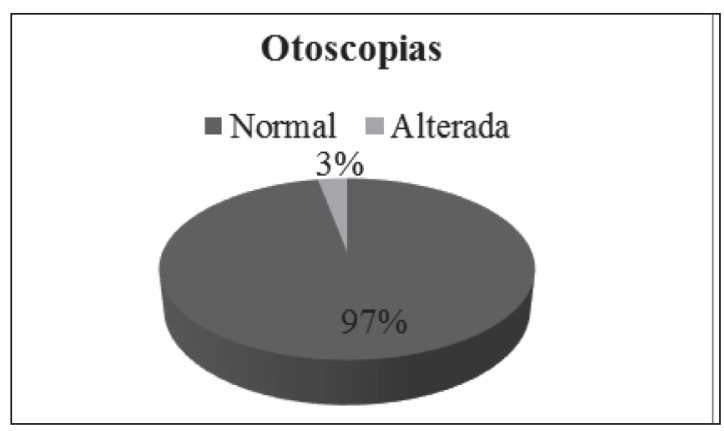

Fonte: Elaboração própria

O tipo de AASI mais comumente indicado foi o retroauricular, seguido pelo intra-auricular e intracanal (Gráfico 5). O AASI tipo microcanal não foi indicado para nenhum paciente. $\mathrm{O}$ circuito aplicado a todos os aparelhos foi de tecnologia digital. Quanto ao molde auricular, o tipo concha foi o mais utilizado (Gráfico 6). Os materiais usados para confecção dos moldes foram o silicone e o acrílico com $181(80,1 \%)$ e $45(19,9 \%)$, respectivamente.

Gráfico 5-Tipos de Aparelhos de Amplificação Sonora Individual (AASI) indicados, por orelha.

\begin{tabular}{|l}
\hline \multicolumn{3}{|c|}{ Tipos de Aparelhos de Amplificação Sonora } \\
Individual \\
$97,30 \%$ \\
\\
\end{tabular}

Fonte: Elaboração própria

Gráfico 6 - Frequência dos tipos de moldes auriculares, por orelha

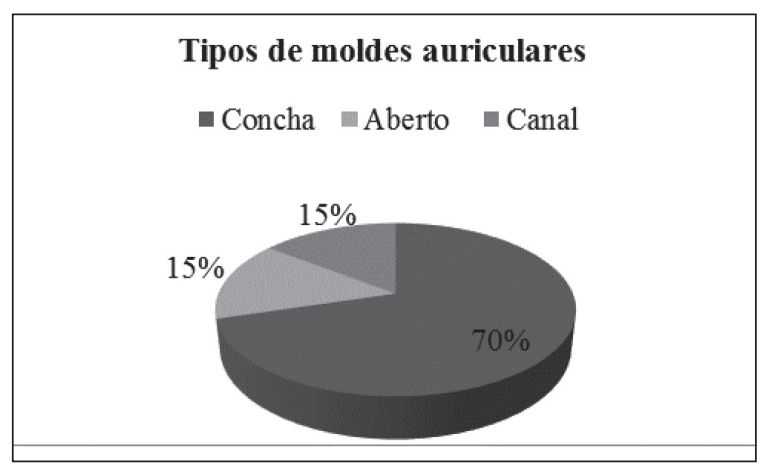

Fonte: Elaboração própria

Foram encontrados 111 (96,5\%) prontuários com reabilitação bilateral e quatro $(3,5 \%)$ com indicação de reabilitação unilateral (mesmo na presença de perda auditiva na orelha contralateral).

\section{DISCUSSÃO}

No contexto da assistência, gestão, e do planejamento das ações nas unidades de saúde, é imprescindível conhecer o perfil da população atendida. Os profissionais envolvidos na prestação de serviço à comunidade necessitam de informações relativas às demandas do serviço em que atuam, haja vista que tal conhecimento pode nortear as decisões e práticas dos atendimentos prestados. Sendo assim, o presente trabalho, levantou informações que podem ser úteis no âmbito do planejamento de novas pesquisas, da gestão e da prática assistencial multiprofissional, no SUS, do Setor de reabilitação auditiva do Centro Especializado em Reabilitação de Salvador, o CEPRED.

Na reabilitação auditiva do CEPRED, o perfil demográfico é compatível com outros estudos, como o de Manzoli (2015), realizada no próprio CEPRED, que encontrou $58,6 \%$ de pacientes do sexo feminino e $53,8 \%$ de idosos, além dos estudos de Gresele et al. (2013) com achados de $52,8 \%$ idosos, e o de Cordeiro (2014), com 60,3\% de pacientes do sexo feminino, em Salvador. A unidade de saúde com essas características em sua demanda deve estar adaptada, pronta para acolher e promover a saúde dessa população. O CER, desse modo, por ser uma referência terciária e de alta complexidade, deve estar atento às necessidades de seus usuários.

Com relação à faixa etária, é importante considerar que a causa mais comum de surdez na espécie humana é a presbiacusia ou "audição do idoso" (GÂNDARA; ALVES, 2017). Trata-se de uma entidade complexa, multifatorial e que se apresenta sob diferentes modos e padrões. A presbiacusia é definida simplificadamente como a perda auditiva tipo sensorioneural bilateral associada ao enveIhecimento, passível de observação a partir dos 30 anos (BICALHO; CINTRA, 2013). Sua prevalência é maior em indivíduos acima de 60 anos (BEVILACQUA et al., 2013). Na presente pesquisa, a perda auditiva sensorioneural bilateral predominou dentre os indivíduos com 60 anos e mais. Indicando a expressiva presença de presbiacusia, nesta população. Em adição, Gresele et al. (2013) também encontraram o quantitativo de $56,2 \%$ deste tipo de PA na população idosa.

A presbiacusia é uma condição com caráter indolente - no início não causa demanda de uso do aparelho auditivo - que começa, em geral, discretamente em frequências altas, fora do espectro da fala habitual, e segue acentuando-se com o avançar da idade, chegando até o comprometimento da percepção dos sons de frequência média e grave. Surge, então, de forma mais premente, a necessidade do recurso de amplificação sonora (GÂNDARA; ALVES, 2017). Essas características podem explicar, em parte, a rejeição ao uso de AASI por alguns pacientes adultos, e a própria não aceitação da perda auditiva ou a falta de percepção da mesma. A propósito, Thodi et al. 
(2013) apontam para a existência de um longo tempo entre o diagnóstico e a protetização, algo em torno de 10 anos

Do ponto de vista demográfico, sabe-se que a sobrevida média das mulheres é maior que a dos homens (IBGE, 2017), tornando-as percentualmente maioria entre os idosos. Soma-se a isso o fato das mulheres serem consideradas mais propensas e atentas ao cuidado de si, em contraposição à relativa postura negligente masculina. Nesse contexto, a predominância de usuários do sexo feminino possivelmente se deve, ao menos em parte, ao fato de as mulheres também tenderem a buscar apoio assistencial quando diante da hipoacusia, como observado no presente estudo e corroborado por outros autores (CORDEIRO, 2014; MANZOLI, 2015). Há indicações, portanto, da necessidade de se fomentar políticas que provoquem mudanças na possível concepção masculina quanto à relevância da sua saúde auditiva. Adicionalmente, deve-se considerar outros fatores relacionados àqueles achados, como a possibilidade de maior prevalência, no sexo feminino, de eventuais doenças que afetam o sistema auditório, como a doença auto-imune da orelha interna, que pode causar distúrbios auditivos e/ou vestibulares em mulheres de meia-idade, na proporção de 2:1, quando se compara ambos os sexos (LOUREÇO; NINA, 2017). Por fim, sabe-se que quanto maior a expectativa de vida de uma população, mais tempo e oportunidades para o sistema auditório adoecer pelas mais diversas causas de perda auditiva, sejam ambientais e/ou intrínsecas ao indivíduo.

A hipoacusia é um fator determinante para a indicação de AASI. Conforme o que é previsto para um centro de saúde especializado em reabilitação auditiva, todos os pacientes referiram a hipoacusia como queixa principal, com uma exceção, na qual o paciente valorizou o zumbido às espessas da redução da acuidade auditiva. Por conseguinte, a avaliação das queixas relativas à orelha interna ou labirinto é uma etapa relevante no atendimento do candidato à reabilitação auditiva. A relação da perda auditiva com outros sintomas otológicos típicos, como zumbido e tontura, pode indicar patologias específicas ou comorbidades, passíveis de tratamento ou reabilitação, como a doença de Ménierè. Neste estudo, o zumbido foi um sintoma típico da orelha interna mais prevalente que a tontura. Consoante com os estudos de Cordeiro (2014) e Manzoli (2015), os quais encontraram a prevalência de $61,5 \%$ e $47,2 \%$ para o zumbido, $51,9 \%$ e $0,9 \%$ para tontura, nesta ordem.

Esse resultado é comum a doenças auditivas, uma vez que a hipoacusia frequentemente vem associada ao zumbido. Isso pode ocorrer, por exemplo, na presbiacusia, no trauma acústico, e nas otites crônicas, dentre outras causas de surdez (DORIGUETO et al., 2017).

Cumpre destacar que ambos, zumbido e tontura, podem ser tão incapacitantes quanto a hipoacusia. A propósito, o zumbido é uma conhecida causa de suicídio, enquanto a tontura pode ser extremamente limitante e causar acidentes como quedas da própria altura. Assim, considerando-se a elevada prevalência desses sintomas, o CER poderia contar com assistência àquelas condições, ou, ao menos, com o apoio de uma rede de referência para atendimento às respectivas queixas, com a devida condução desses casos. Do ponto de vista semiológico, o CER poderia disponibilizar a realização de acufenometria para melhor avaliação das características do zumbido.

Quanto a reabilitação deste sintoma, no CEPRED, se deu apenas pela amplificação do AASI. A referida instituição não o disponibiliza com gerador de ruído, o que poderia diminuir a proeminência do zumbido por meio da dissimulação, reduzindo a atenção ao mesmo e o incômodo (IMLAU, 2015; SANTOS et al., 2012). Desta forma, o CER deveria levar essas demandas em consideração, disponibilizando acesso a esses tipos de dispositivos. Quanto à tontura, exigem-se recursos mais amplos para o diagnóstico otoneurológico através de métodos equilibrométricos e nistagmográficos, para uma abordagem medicamentosa, e para a reabilitação vestibular em unidades de referência. Até o presente momento, a reabilitação vestibular e do zumbido não são contempladas pela proposta do CER no contexto do SUS. Deste modo, considerando-se a elevada prevalência de zumbido e tontura entre pacientes com perda auditiva, por exemplo, defendemos a extensão de serviços do CER para atendimento a essas necessidades.

No CER, a avaliação do candidato ao uso de AASI inclui o exame clínico otorrinolaringológico. Nesta abordagem, a otoscopia representa uma etapa crítica, pois auxilia no diagnóstico de doenças otológicas com repercussão auditiva e prevê possíveis perdas auditivas do tipo condutiva documentadas a posteriori pela audiometria. Além disso, condições transitórias como rolha de cera e otites externas podem ser diagnosticadas e tratadas antes dos procedimentos audiológicos. Os pacientes incluídos no presente trabalho apresentaram otoscopia normal. Essa descrição está em consonância com o perfil dos indivíduos analisados aqui, uma vez que a perda auditiva sensorioneural e a presbiacusia, cuja prevalência é significativa entre idosos, é uma condição que não se relaciona a alterações significativas da membrana timpânica. A respeito dos achados otoscópicos, somente as orelhas com membranas timpânicas perfuradas exigiram um cuidado especial no sentido de evitar acidente com a massa de pré-moldagem auricular.

A documentação da perda auditiva e a determinação de suas características através da audiometria e demais exames audiológicos, quando indicados, constituem etapa crucial na avaliação do candidato à reabilitação auditiva. No presente estudo, a maioria dos pacientes apresentou perda auditiva bilateral, sendo equivalente aos achados de Manzoli (2015), em que demonstraram 95,8\% de perda com esta característica. Dessa forma, é possível perceber que indivíduos com perdas auditivas bilaterais buscam mais pelo Centro Especializado em Reabilitação, indicando que este tipo de perda provoca maior necessidade de uso do AASI. Enquanto a presença de limiares auditivos melhores ou normais em uma orelha, pode compensar e 
mascarar uma possível PA na orelha contralateral, reduzindo ou extinguindo a busca pela reabilitação.

O tipo de perda auditiva mais comum foi a sensorioneural, corroborando com pesquisas prévias que encontraram prevalência de 73,0\% (MANZOLI, 2015) e 73,12\% (GRESELE et al., 2013). Embora a perda auditiva do tipo condutiva apresente melhor prognóstico para a reabilitação com AASI, muitos casos são passíveis de tratamento, seja clínico ou cirúrgico, ou são condições transitórias, o que reduz a frequência de indicação de AASI (PEREIRA, 2015). Por outro lado, a perda auditiva tipo sensorioneural geralmente só é passível de reabilitação por prótese auditiva, com exceção dos casos de surdez súbita, nos quais se indica uso de medicações, inicialmente, ou nas perdas severas e profundas, em que se pode lançar mão da cirurgia de implante coclear.

Quanto ao tipo de AASI, o mais indicado foi o retroauricular (Behind-The-Ear/BTE), equiparando-se aos estudos de Zandavalli et al. (2009) e Araujo et al. (2011), usados em $71,4 \%$ e $88,2 \%$ dos pacientes, respectivamente. No setor público de saúde auditiva, onde se aplica a licitação, prioriza-se a relação custo-benefício. Assim, a preferência pelo BTE pode ser explicada por diferentes motivos. Trata-se de um AASI versátil, com possibilidade de adaptação em diversos tipos e graus de PA (PEREIRA, 2015), manutenção facilitada e menos custosa para o próprio paciente, que leva em consideração a maior durabilidade da bateria elétrica, cujo tamanho é maior. Outra vantagem é o fácil manuseio pelos pacientes, vez que a população idosa predominante neste estudo - tende a dispor de menor destreza manual e menor acuidade visual (PIZA, 2017). A colocação e a retirada do AASI, a limpeza adequada, e o controle de volume são mais fáceis de realizar com o retroauricular quando comparado aos intra-aurais, os quais requerem maiores cuidados e habilidade.

Os aparelhos intra-aurais, sendo eles os intra-auriculares (In-The-Ear/ITE), intracanais (In-The-Canal/ITC) e microcanais (Completely-In-Canal/CIC), tiveram menor frequência de indicação ou não foram indicados, como visto com o CIC. Esses aparelhos intra-aurais não têm potência para amplificar perdas grandes, o que limita as adaptações em perdas severas e profundas (PEREIRA, 2015; PIZA, 2017; TEIXEIRA; GARCEZ, 2015). Suas indicações são feitas observando-se, sobretudo, o grau e a configuração da perda auditiva. Assim, geralmente são adaptados em perdas de configuração plana e de graus leve a moderado. Ficam limitados também na perda auditiva do tipo em rampa, pois faz com que o paciente sinta desconforto referente a ruídos por conta da preservação dos limiares nas frequências graves. Em acréscimo, as oreIhas com membrana timpânica perfurada e/ou associadas à otorreia frequente também limitam o uso de AASI ITC e $\mathrm{CIC}$, pois a umidade relacionada a estas condições pode danificar o circuito eletrônico, bem como piorar a infecção pelo "tamponamento" do MAE. Esses usam baterias menores que duram menos e exigem habilidade manual para manejo. Eles têm como vantagem uma melhor estética, pois são discretos, permitindo maior aceitação da prótese auditiva pelos usuários.

No que tange ao tipo de tecnologia aplicada a esses aparelhos disponibilizados pelo SUS, foi possível verificar concordância com o estudo de Zandavalli et al. (2009), o qual demonstrou percentagem idêntica. Os circuitos híbridos praticamente não se encontram disponíveis no mercado, e o analógico perde cada vez mais sua participação, em decorrência do aumento da acessibilidade e vantagens dos aparelho digitais (PIZA, 2017).

O molde de silicone foi o mais utilizado. Por ser um material macio, flexível, confortável, hipoalérgico e que proporciona maior vedação e aceitação, é, em geral, o de escolha. Contudo, não é possível realizar muitas modificações acústicas e estéticas, pois é de difícil perfuração. $\mathrm{O}$ acrílico, por ser mais rígido, oferece o risco de lesões na orelha externa, são quebráveis e têm indicações mais específicas. Na presença de infecções otológicas crônicas ou recorrentes, o molde a ser escolhido deve ser de fácil limpeza e manutenção, dando-se preferência aos de acrílico por apresentarem menor retenção de impurezas, além de ser facilmente laváveis (IWAHASHI et al., 2011).

O molde mais comum foi o tipo concha, ao passo que o restante dos casos foi equitativamente dividido entre os tipos aberto e canal. No estudo de Zandavalli et al. (2009), o molde concha foi encontrado em $35,7 \%$ dos pacientes. Segundo Pereira (2015), este tipo de molde apresenta amplas vantagens, proporcionando maior vedação acústica, impedindo microfonia, além de incrementar o ganho do AASI. Sua indicação ocorre sobretudo quando a perda auditiva requer um aparelho com potência elevada. Por sua vez, o molde tipo canal é preferencialmente indicado para perdas auditivas de grau leve e moderado, enquanto o tipo aberto pode ser usado quando a ventilação torna-se insuficiente e abrange um espectro de frequência e intensidade menores. Embora o presente trabalho não tenha levantado informações sobre o grau de perda auditiva, a maior prevalência do molde tipo concha sugere uma relevante demanda de potência de amplificação sonora. Neste sentido, espera-se que indivíduos com perdas auditivas maiores busquem atendimento nas unidades de saúde por conta da repercussão negativa em sua vida social (CORDEIRO, 2013; IÓRIO, 2015).

Gresele et al. (2013) e Araujo et al. (2011) encontram $82,6 \%$ de adaptação bilateral, dos 1572 prontuários estudados, e $86,1 \%$ dos 440 indivíduos pesquisados, respectivamente, equiparando aos achados do presente estudo. De acordo com Mueller et al. (2014), na presença de perda auditiva em ambas as orelhas, a adaptação bilateral deve ser a opção de escolha, pois as vantagens são extremamente significantes. Esse tipo de adaptação possibilita efeito de somação, eliminação do efeito sombra da cabeça, melhora da localização sonora e reconhecimento da fala no ruído, bem como da qualidade sonora e do balanço espacial.

$\mathrm{Na}$ adaptação unilateral com perda auditiva em ambas as orelhas, é conhecido o risco de desencadeamento 
da privação sensorial, ou seja, depois de algum tempo da amplificação sonora unilateral, há uma piora no reconhecimento de fala na orelha contralateral sem que haja piora dos limiares auditivos. Assim, de acordo com a Portaria SAS/MS no 587 de 2004, esse tipo de adaptação deve ser concretizada nas seguintes situações: perda auditiva assimétrica e a avaliação indicar o não benefício da adaptação bilateral ou com um dos lados anacúsico; perda auditiva bilateral, quando as condições anatômicas e/ou fisiológicas da orelha externa e/ou média impossibilitarem a utilização de AASI; desconforto em sua utilização por condução óssea; e quando for opção do paciente após experiência bilateral.

No entanto, o estudo de Zandavalli et al. (2009), realizado em 14 centros auditivos da rede privada, na cidade de Porto Alegre, Rio Grande do Sul, revela a predominância de adaptação unilateral (78,6\%), justificada pela realidade financeira da população de países como o Brasil. O AASI não possui preços acessíveis a determinadas classes sociais, favorecendo a adaptação de forma contraindicada. Ressalta-se, nesse sentido, a importância de políticas públicas de saúde, como o CER, um dos grandes responsáveis pelo processo de reabilitação auditiva gratuita através do SUS, que prioriza e efetiva a adaptação bilateral de AASI.

Não encontramos, na literatura, estudo semelhante realizado em outro CER. Por outro lado, o presente trabalho, de caráter descritivo e exploratório, teve como importantes limitações o pequeno tamanho da amostra e a ausência da pesquisa sobre o grau e a configuração da perda auditiva de cada caso estudado. Assim, estudos mais abrangentes e envolvendo maior número de pacientes poderiam ser realizados em outros CER.

\section{CONCLUSÃO}

A partir deste estudo descritivo e exploratório, pode-se constatar que, no Setor Auditivo do CER de referência do Estado da Bahia, em Salvador, a maioria dos pacientes atendidos são idosos e do sexo feminino, com predominância de perda auditiva do tipo sensorioneural bilateral e com otoscopias normais. Esses achados sugerem potencial importância da presbiacusia. Deste modo, uma atenção especial deve ser voltada no sentido de acolher e promover a saúde de idosos e, em particular, do sexo feminino. Por outro lado, esse perfil demográfico indica que homens devem ser esclarecidos e encorajados a buscar assistência à saúde auditiva. Além da hipoacusia, motivo da reabilitação auditiva, os pacientes apresentaram elevada prevalência de queixas de zumbido e tontura, os quais demonstram a necessidade de extensão assistencial a essas demandas, seja no próprio CER ou através do apoio de uma rede de referência para a devida condução desses casos.

$\mathrm{Na}$ reabilitação auditiva promovida pelo CER, confirma-se a efetiva e indispensável adaptação bilateral quando na presença de hipoacusia em ambas orelhas. 0 tipo de AASI mais indicado foi o retroauricular, possivel- mente motivado pela necessidade de uma boa relação custo-benefício do setor público e do próprio paciente, pela versatilidade desse aparelho, e para atender às necessidades do público idoso. De igual modo, o molde tipo concha possibilita melhores condições para o AASI de grande ganho acústico, em sua maior parte confeccionado a partir do silicone flexível, melhor tolerado e vantajoso em muitos aspectos. A tecnologia dos AASIs foi exclusivamente digital.

Estudos futuros, no CEPRED e em outros CER, envolvendo um maior número de indivíduos e baseados em coleta detalhada de múltiplas informações, podem auxiliar a determinar com acurácia as características da população atendida no Setor Auditivo do CEPRED, bem como da assistência oferecida pela instituição. Deste modo, poder-se-á obter um perfil do usuário e do serviço oferecido, levantando informaç̧̃̃es e questões sobre doenças, prevenção, oferta de serviços e planejamento estratégico.

\section{REFERÊNCIAS}

ARAUJO, T. M. et al. Pronto atendimento a usuários de dispositivo de amplificação sonora. Rev Soc Bras Fonoaudiol, v. 16, n. 4, p. 466-473, 2011. Disponível em: www.scielo.br/pdf/rsbf/v16n4/v16n4a17.pdf. Acesso em: 31 dez. 2016.

BEVILACQUA, M. C. et al. Survey of hearing disorders in an urban population in Rondonia, Northern Brazil. Rev. Saúde Pública, São Paulo, v. 47, n. 2, p. 309-315, Apr. 2013. Disponível em: www.scielo.br/scielo. php?script=sci_arttext\&pid=S0034-89102013000200309. Acesso em: 19 nov. 2017.

BICALHO, M. A. C.; CINTRA, M. T. G. Modificações fisiológicas sistêmicas no envelhecimento. In: MALLOY-DINIZ, L. F. et al. Neuropsicologia do envelhecimento - uma abordagem multidimensional. Porto Alegre: Artmed, 2013. cap. 2.

BRASIL. IBGE. Agência IBGE Notícias. 2017. Disponível em: agenciadenoticias. ibge.gov.br/agencia-noticias/2013-agenciadenoticias/releases/18263-pnad-2016-populacao-idosa-cresce-16-0frente-a-2012-e-chega-a-29-6-milhoes.html. Acesso em: 08 jun. 2018.

BRASIL. Portaria no 2.073, de $\mathbf{2 8}$ de setembro de 2004. Institui a Política Nacional de Atenção à Saúde Auditiva. Brasília, DF, Ministério da Saúde, 2004. Disponível em: bvsms.saude.gov.br/bvs/saudelegis/gm/2004/ prt2073_28_09_2004.html. Acesso em: 03 fev. 2018.

BRASIL. Portaria no 587, de 07 de outubro de 2004. Dispõe sobre a organização e a implantação das Redes Estaduais de Atenção à Saúde Auditiva. Brasília, DF, Ministério da Saúde, 2004. Disponível em: bvsms. saude.gov.br/bvs/saudelegis/sas/2004/prt0587_07_10_2004.html. Acesso em: 03 fev. 2018.

CORDEIRO, B. B. Perfil epidemiológico e clínico-audiológico dos pacientes do setor de audiologia de um serviço público de fonoaudiologia de salvador em 2013. 2014. 76 f. Dissertação (Mestrado em Processos Interativos dos Órgãos e Sistemas) -Instituto de Ciências da Saúde, Universidade Federal da Bahia, Salvador, 2014. Disponível em: repositorio.ufba.br/ri/bitstream/ri/17935/1/CORDEIRO\%2c\%20 Bianca\%20Bastos.pdf. Acesso em: 19 nov. 2017.

DORIGUETO, R. S. et al. Semiologia dos órgãos da audição e do equilíbrio. In: PIGNATARI, S. S. N.; ANSELMO-LIMA, W.T. (Org.) Tratado de Otorrinolaringologia. 3. ed. Rio de Janeiro: Elsevier Editora Ltda, 2017.

FERRITE, S. et al. Validity of self-reported hearing loss in adults: 
performance of three single questions. Rev. Saúde Pública, São Paulo, v. 45 , n. 5, p. 824-30, 2011. Disponível em: www.scielo.br/scielo. php?script=sci_Abstract \&pid $=$ S0034-89102011005000050\&lng $=$ pt\&n rm=iso\&tlng=en. Acesso em: 25 maio 2018.

GÂNDARA, M. E. R.; ALVES, F. R. A. Presbiacusia. In: PIGNATARI, S. S. N; ANSELMO-LIMA, W. T. (Org.) Tratado de otorrinolaringologia. 3. ed. Rio de Janeiro: Elsevier Editora Ltda, 2017.

GRESELE, A. D. P. et al. Levantamento e análise de dados de pacientes atendidos em um programa de concessão de aparelhos de amplificação sonora individual. CoDAS, São Paulo, v. 25, n. 3, p.195-201, 2013. Disponível em: www.scielo.br/pdf/codas/ v25n3/02.pdf. Acesso em: 02 dez. 2017.

IMLAU, D. Análise do benefício de tratamento de zumbido com geradores de ruído: estudo de caso. 2015. 29 f. TCC (Curso de Especialização em Audiologia Clínica) -Universidade Tuiuti do Paraná, Curitiba, 2015.

IÓRIO, M. C. M. Qualidade de vida intervenção fonoaudiológica por meio da adaptação de próteses auditivas. In: BOÉCHAT, E. M. et al. (Org.) Tratado de Audiologia. 2. ed. Rio de Janeiro: Guanabara KOOGAN, 2015. cap. 41.

IWAHASHI, J. H. et al. Protocolo de Seleção e Adaptação de Prótese Auditiva para Indivíduos Adultos e Idosos. Arq. Int. Otorrinolaringol, São Paulo, v. 15, n. 2, p. 214-222, abr./maio/jun. 2011. Disponível em: www.scielo.br/pdf/aio/v15n2/a15v15n2.pdf. Acesso em: 07 fev. 2018.

LOUREÇO, E. A; NINA, L. D. Doença Imunomediada da Orelha Interna. In: PIGNATARI, S. S. N; ANSELMO-LIMA, W.T.(Org). Tratado de otorrinolaringologia. 3. ed. Rio de Janeiro: Elsevier Editora Ltda, 2017.

MANZOLI, G. N. Caracterização demográfica, clínica e genética da deficiência auditiva na Bahia. 2015. 129 f. Tese (Doutorado em Patologia Humana )- Faculdade de Medicina, Universidade Federal da Bahia, Fundação Oswaldo Cruz, Centro de Pesquisas Gonçalo Moniz, Salvador, 2015.

MUELLER, H.G. et al. Hearing aid stules and fitting aplcations. In: Modern hearing aids: pre-fitting testing and selection considerations.
San Diego: Plural Publishing, 2014. cap. 7.

PAGNOSSIM, D. F. et al. O processo de seleção, indicação e adaptação de Aparelhos de Amplificação Sonora Individual: como fazemos? In: BELEN, S. A. et al.(Orgs.) Saúde Auditiva: da teoria à prática. São Paulo: Editora Santos, 2010. cap. 8.

PEREIRA, R. C. Molde Auricular. In: Prótese Auditiva. Rio de Janeiro: Revinter, 2015. cap. 3.

PIZA, M. R. T. Aparelho de Amplificação Sonora Individual: aspectos de ordem prática para o Otorrinolaringologista. In: PIGNATARI, S. S. N.; ANSELMO-LIMA, W.T. (Org.) Tratado de Otorrinolaringologia. 3. ed. Rio de Janeiro: Elsevier, 2017.

SANTOS, G. M. et al. A utilização de prótese auditiva genéricas com geradores de som integrado para controle do zumbido: estudo piloto. Arq. Int. Otorrinolaringol, São Paulo, v. 16, n. Suppl. 1., maio 2012. Disponível em: arquivosdeorl.org.br/additional/ acervo_port. asp?id=945. Acesso em: 03 fev. 2018.

SILVA, T. F. et al. Política e gestão na atenção à saúde auditiva no estado da Bahia: a experiência do Centro Estadual de Prevenção e Reabilitação de Deficiências (CEPRED). In: BEVILACQUA, M. C. et al. (Org.) Saúde auditiva no Brasil: políticas, serviços e sistemas. São José dos Campos: Pulso Editorial, 2010. cap. 11.

TEIXEIRA, A. R.; GARCEZ, V. Aparelho de Amplificação Sonora Individual: Componentes e Características Eletroacústicas. In: BOÉCHAT, E. M. et al. (Org.). Tratado de Audiologia. 2. ed. Rio de Janeiro: Guanabara KOOGAN, 2015. cap 33. p. 253-258.

THODI, C. et al. Adult hearing screening: follow-up outcomes. Am. j. audiol., Rockville, v. 22, n. 1, p. 138-185, 2013.

ZANDAVALLI, M. B. et al. Rotina de procedimentos utilizados na seleção e adaptação de aparelhos de amplificação sonora individual em centros auditivos na cidade de Porto Alegre, Brasil - RS. Rev CEFAC, São Paulo, v. 11, Supl.1, p. 106-115, 2009. Disponível em: www.scielo.br/pdf/rcefac/ v11s1/07-08.pdf. Acesso em: 01 dez. 2016.

Submetido em: 19/07/2019

Aceito em: 02/01/2020 